\title{
Inmovilización microbiana en polímeros sintéticos para el tratamiento de aguas residuales
}

\author{
Microbial immobilization on synthetic polymers for wastewater treatment
}

Wilmar Alirio Botello Suárez, Juan Camilo Ortiz Varón, Sergio Andrés Peña Perea.

\section{Resumen}

Objetivo. Evaluar la capacidad de inmovilización microbiana de algunos polímeros sintéticos, y establecer su desempeńo durante la partida y operación de un reactor biológico híbrido (RBH), aplicado al tratamiento de aguas residuales domésticas. Métodos. Para la evaluación fueron seleccionados cinco polímeros: tereftalato de polietileno (PET), polibutadieno (BD), espuma de poliuretano (EPU), poliestireno expandido (EPS) y polietileno (P). Los materiales con mayor capacidad de inmovilización (expresado como la cantidad de unidades formadoras de colonias contenidas en un gramo de material, $\mathrm{UFCg}^{-1}$ ), fueron incorporados a un $\mathrm{RBH}$, con volumen operacional de $10,4 \mathrm{~L}$, alimentado con agua residual sintética. La eficiencia del sistema fue establecida en términos de remoción de la materia orgánica contenida en el afluente durante 13 días de operación continua. Resultados. Los polímeros que presentaron mayor capacidad de inmovilización fueron: $\mathrm{BD}\left(2,2 \times 10^{7} \mathrm{UFCg}^{-1}\right)$ y EPU $\left(4,6 \times 10^{7} \mathrm{UFCg}^{-1}\right)$. El $\mathrm{RBH}$, sometido a una carga orgánica volumétrica de $2,7 \mathrm{Kg} \mathrm{DQO} \mathrm{m}^{-3} \mathrm{~d}^{-1}$, presentó estabilidad operativa en todos los tratamientos evaluados, alcanzando una remoción de materia orgánica de 78\% y 94\%, al emplear BD y EPU como soportes de inmovilización, respectivamente.

Palabras Clave. Agua residual doméstica, soportes de inmovilización, reactor biológico híbrido.

\section{Abstract}

Objective. Evaluate the ability of microbial immobilization of several synthetic polymers, and establish its performance during the start-up and operation of a hybrid biological reactor (HBR), applied to domestic wastewater treatment. Methods. For evaluation we selected five polymers: polyethylene terephthalate (PET), polybutadiene (BD), polyurethane foam (PUF), expanded polystyrene (EPS) and polyethylene (PE). Materials with higher immobilization capacity (expressed as the number of colony forming units contained in one gram of material, $\mathrm{CFUg}^{-1}$ ) were added to a $\mathrm{HBR}$, with operational volume of 10,4L, fed with synthetic wastewater. The efficiency of the system was established in terms of removal of organic matter contained in the effluent for 13 days of continuous operation. Results. Polymers showing the high immobilization capacity were: $\mathrm{BD}\left(2,2 \times 10^{7} \mathrm{CFUg}^{-1}\right)$ and PUF $\left(4,6 \times 10^{7}\right.$ $\left.\mathrm{CFUg}^{-1}\right)$. The HBR, subjected to organic loading rate of $2.7 \mathrm{~kg} \mathrm{COD} \mathrm{m}^{-3} \mathrm{~d}^{-1}$, showed operational stability for the treatments evaluated, reaching a removal of organic matter of $78 \%$ and $94 \%$, by using $\mathrm{BD}$ and PUF as immobilization supports, respectively.

Keywords. Domestic wastewater, hybrid biological reactor, immobilization supports. 


\section{Introducción}

La afectación de sistemas hídricos es una de las principales problemáticas generadas como consecuencia del crecimiento poblacional y la industrialización. Diariamente es producido un importante volumen de efluentes domésticos con elevado potencial contaminante, siendo de interés prioritario desarrollar y optimizar sistemas que permitan, además de mitigar los efectos adversos que ocasiona su vertimiento, atender las disposiciones establecidas en la normatividad ambiental. En este escenario, el uso de procesos biológicos surge como una importante alternativa para el tratamiento de efluentes, la cual aprovecha las capacidades metabólicas de la biomasa microbiana asociada al sistema, para reducir, de forma significativa, la concentración de sólidos en suspensión, materia orgánica y elementos tales como nitrógeno y fósforo $(1,2)$.

El desarrollo de reactores biológicos híbridos (RBH), ha permitido generar unidades de tratamiento compactas, las cuales alcanzan altos niveles de eficiencia y presentan un desempeño más estable que los sistemas convencionales $(3,4)$. La configuración de estos reactores permite incrementar el tiempo de retención celular y la concentración la biomasa asociada al proceso (fija y en suspensión), lo cual posibilita aplicar altas cargas orgánicas $(5,6)$. Esto se logra a través de un mecanismo de inmovilización pasiva de la biomasa sobre un material soporte, lo cual posibilita la generación de una biopelícula, estructura que presenta una alta actividad microbiana, que al entrar en contacto con el efluente permite alcanzar elevadas tasas de biodegradación (7). No obstante, el éxito de la implementación de un RBH requiere la selección de un material de soporte idóneo, el cual garantice la generación, mantenimiento y viabilidad de una biopelícula metabólicamente activa.

Algunos criterios para la selección de soportes se basan en el cumplimiento de diversos parámetros, entre ellos, presencia de una superficie amplia para la adherencia celular, facilidad de operación y regeneración, porosidad (con el fin de permitir un intercambio constante de materiales), estabilidad química y biológica, y resistencia a enzimas, solventes y a cambios de presión, entre otros $(7,8)$. Teniendo en cuenta estas características, diversas investigaciones han analizado el desempeño de materiales soporte sometidos a diferentes configuraciones operacionales en reactores de película fija convencionales $(9,10)$. Al respecto, han sido empleados un gran número de polímeros sintéticos, entre ellos, espuma de poliuretano $(11,12)$, cloruro de polivinilo y polipropileno (13), los cuales atienden a las características mencionadas anteriormente, y cuya implementación ha permitido alcanzar diferentes grados de eficiencia en procesos de tratamiento. Consecuentemente, dada la gran variedad de polímeros sintéticos existentes, muchos de los cuales constituyen componentes mayoritarios de una amplia gama de productos descartables de uso cotidiano, determinar su capacidad para la inmovilización de biomasa microbiana representa una interesante estrategia de aprovechamiento alternativo de dichos materiales, optimizando el desempeño de sistemas de tratamiento biológico de aguas residuales.

Por lo anteriormente expuesto, el presente trabajo tuvo como objetivo evaluar la eficiencia de diversos polímeros sintéticos en función a su capacidad de inmovilización de biomasa microbiana, y determinar su desempeño en términos de remoción de materia orgánica, durante la partida y operación de un $\mathrm{RBH}$ alimentado con aguas residuales domésticas.

\section{Materiales y métodos}

Selección de soportes. La inmovilización microbiana fue evaluada sobre los siguientes polímeros sintéticos: i. tereftalato de polietileno (PET), ii. caucho sintético (polibutadieno, BD), iii. espuma de poliuretano (EPU), iv. poliestireno expandido (EPS) y v. polietileno (P). El tamaño de partícula para cada material fue de $0,2 \mathrm{~cm}^{3}$ (PET y P) y 1,0 $\mathrm{cm}^{3}$ (EPU, EPS y BD). Antes de su uso todos los soportes fueron esterilizados en autoclave $\left(121^{\circ} \mathrm{C}\right.$ por $20 \mathrm{~min}$ ). Los ensayos fueron establecidos en 
matraces conteniendo $200 \mathrm{~mL}$ de caldo Luria Bertani (LB) con 10 partículas de material soporte, $y$ fueron inoculados al $2 \%\left(\mathrm{OD}_{600}=0,2\right)$ con el aislado ambiental Bacillus sp. H22, caracterizado previamente por el grupo de investigación (14). El tiempo de incubación fue de $24 \mathrm{~h}$, a una temperatura de $30 \pm 2^{\circ} \mathrm{C}$ y agitación constante a 150 r.p.m. Para la evaluación de la capacidad de inmovilización microbiana, cada $12 \mathrm{~h}$ fue retirada del ensayo una partícula de material soporte de cada uno de los montajes, y lavada con solución salina estéril en condiciones asépticas. Posteriormente, las partículas fueron suspendidas en $9 \mathrm{~mL}$ de agua peptona y agitadas vigorosamente mediante vórtex por un minuto. Las concentración de biomasa presente en la suspensión generada, la cual representa la biomasa total retenida por cada partícula, fue cuantificada mediante establecimiento de diluciones seriadas y siembra en superficie en agar LB, y expresada como unidades formadoras de colonia por gramo de soporte $\left(\mathrm{UFCg}^{-1}\right)$. Por otra parte, fue calculada la densidad microbiana suspendida en el medio, a través de la medición de la densidad óptica del cultivo $\left(\mathrm{OD}_{600}\right)$ durante $12 \mathrm{~h}$ de proceso. Todos los ensayos fueron realizados por triplicado. Los soportes más eficientes fueron seleccionados por medio de análisis de varianza mediante el test de Tukey, con un nivel de significancia de $1 \%$, en función de su capacidad de inmovilización en el periodo de tiempo establecido, y fueron empleados en los análisis posteriores.

Configuración del sistema. Fue realizada la evaluación del desempeño de un reactor biológico híbrido $(\mathrm{RBH})$, establecido a escala de laboratorio, y adaptado para contener los medios soportes seleccionados ( $40 \%$ de su volumen útil). El sistema implementado se ilustra en la Figura 1, y fue conformado por una cámara de reacción de 10,4L, provista de los polímeros seleccionados (medio soporte), y un módulo de sedimentación de 11,8L. Al reactor fue acoplado un dispositivo de inyección de aire (filtrado mediante membrana de nitrocelulosa de $0,45 \mu \mathrm{m})$, con flujo de 4,6 $\mathrm{Lmin}^{-1}$, el cual sirvió tanto para el suministro de oxígeno, como para la agitación del licor de mezcla. La partida del sistema fue realizada en modo discontinuo con un tiempo operacional de tres días. Para ello, fue empleado un volumen operacional de $20 \mathrm{~L}$ de sustrato (agua residual sintética), y posteriormente fue realizada la inoculación con la suspensión bacteriana anteriormente mencionada $\left(2 \%, \mathrm{OD}_{600}=0,2\right), \mathrm{La}$ operación fue desarrollada a temperatura ambiente $\left(27,5 \pm 1^{\circ} \mathrm{C}\right)$. Terminado el periodo de partida, el sistema fue alimentado con flujo continuo $\left(\mathrm{Q}=3,96 \mathrm{Lh}^{-1}\right)$ durante 10 días. Este procedimiento fue realizado separadamente para cada uno de los soportes seleccionados. Adicionalmente, fue dispuesto un ensayo control desprovisto de material soporte.

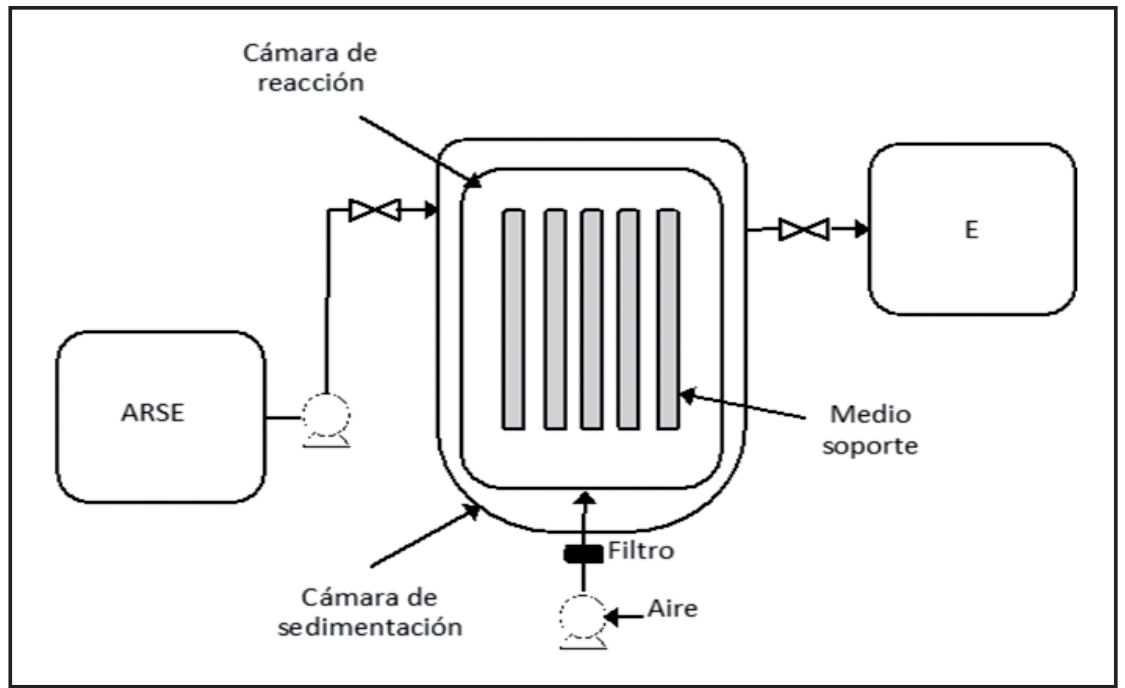

Figura 1. Configuración del reactor biológico híbrido (RBH). ARS: Afluente (Agua residual sintética); E: Efluente. 
Agua residual. El reactor fue alimentado con agua residual sintética (ARS), preparada con la finalidad de simular las propiedades químicas presentes en un agua residual doméstica típica. En su preparación fueron empleados los siguientes componentes $\left(\mathrm{mgL}^{-1}\right)$ : leche en polvo 102,0; detergente 3,0; gelatina 34,0 (compuesta por colágeno 8,5, sales minerales 0,2 y $\left.\mathrm{H}_{2} \mathrm{O} \quad 1,3\right)$; almidón soluble 171,0; $\mathrm{MgSO}_{4} \cdot 7 \mathrm{H}_{2} \mathrm{O} \quad 3,0$; $\mathrm{KH}_{2} \mathrm{PO}_{4} 44,0 ;\left(\mathrm{NH}_{4}\right)_{2} \mathrm{SO}_{4} 72,4, \mathrm{NaHCO}_{3} 150,0$. $\mathrm{El}$ agua obtenida fue almacenada a $-5^{\circ} \mathrm{C}$ hasta su uso. En la Tabla 1 son descritas las características físico-químicas del sustrato generado.

Tabla 1. Características físico-químicas del agua residual sintética empleada

\begin{tabular}{|cc|}
\hline Parámetro & ARS \\
\hline $\mathrm{pH}$ & $7,15 \pm 0,55$ \\
\hline Conductividad eléctrica $(\mathrm{mS})$ & $730,6 \pm 66,8$ \\
\hline Sólidos disueltos totales $\left(\mathrm{mgL}^{-1}\right)$ & $391,7 \pm 6,1$ \\
\hline DBO $\left(\mathrm{mLL}^{-1}\right)$ & $139,5 \pm 54,6$ \\
\hline DQOt $\left(\mathrm{mgL}^{-1}\right)$ & $296,7 \pm 87,2$ \\
\hline
\end{tabular}

DBO: demanda bioquímica de oxígeno; DQO Total: demanda química de oxígeno total.

Métodos analíticos. Un volumen de 1L de muestra del afluente y efluente del RBH fue colectado a los 0, 8 y 13 días de operación, con la finalidad de determinar los parámetros de demanda química de oxígeno total (DQO total) y demanda bioquímica de oxígeno (DBO). La eficiencia de remoción de materia orgánica $(\% \mathrm{R})$ fue calculada mediante la siguiente ecuación:

$$
\% R=\frac{C_{0}-C_{1}}{C_{0}} \times 100
$$

donde $\mathrm{C}_{0}$ y $\mathrm{C}_{1}$ son las concentraciones de $\mathrm{DBO}$ ó DQO total presentes en el afluente y efluente del $\mathrm{RBH}$, respectivamente. La estabilidad del proceso fue analizada diariamente, mediante determinación de los siguientes parámetros: $\mathrm{pH}$, conductividad eléctrica, temperatura, y sólidos disueltos totales (SDT). Todas las determinaciones fueron realizadas conforme a los métodos descritos por APHA, AWWA, WPCF 2005 (15).

\section{Resultados}

\section{Crecimiento de biomasa y selección de soportes}

El perfil cinético de crecimiento bacteriano realizado a partir del sobrenadante de cada uno de los ensayos establecidos (biomasa en suspensión) se relaciona en la Figura 2. Dadas las características de este perfil, se determinó el final de la fase de crecimiento exponencial para cada una de las pruebas en un periodo de $12 \mathrm{~h}$.



Figura 2. Perfil cinético de la biomasa en suspensión en cada uno de los ensayos establecidos. EPS: poliestireno expandido; PET: tereftalato de polivinilo, EPU: espuma de poliuretano; BD: polibutadieno; P: polietileno. 
La Figura 2 relaciona el perfil cinético determinado en la biomasa en suspensión hasta el final de la fase de crecimiento exponencial $(T=12 \mathrm{~h})$, el cual fue el periodo considerado para la cuantificación de microorganismos adheridos a los soportes evaluados. La concentración celular retenida por cada soporte se relaciona en la Tabla 2.

Tabla 2. Valores medios de la concentración celular retenida $\left(\mathrm{UFCg}^{-1}\right)$ en cada uno de los soportes evaluados estimados al final de la fase de crecimiento exponencial.

\begin{tabular}{|cc|}
\hline Soporte & UFCg-1 Soporte \\
\hline EPU & $4,6 \times 107 \mathrm{a}$ \\
\hline BD & $2,2 \times 107 \mathrm{~b}$ \\
\hline EPS & $1,4 \times 105 \mathrm{c}$ \\
\hline PET & $2,2 \times 105 \mathrm{c}$ \\
\hline P & $2,1 \times 105 \mathrm{c}$ \\
\hline
\end{tabular}

Valores seguidos por letras minúsculas diferentes presentan diferencias significativas (Tukey a $1 \%$ de probabilidad: $p<0,01)$. EPS: poliestireno expandido; PET: tereftalato de polivinilo, EPU: espuma de poliuretano; BD: polibutadieno; P: polietileno.
Los polímeros con mejor retención fueron la espuma de poliuretano $\left(4,6 \mathrm{x}^{7} \mathrm{UFCg}^{-1}\right)$ y el polibutadieno $\left(2,2 \times 10^{7} \mathrm{UFCg}^{-1}\right)$. Los demás soportes presentaron retención del orden de $10^{5} \mathrm{UFCg}^{-1}$ y no fueron considerados para la realización de análisis posteriores, al presentar diferencias significativas respecto a los soportes con retención más eficientes (Tukey al $1 \%$ de probabilidad: $p<0,01$ ).

\section{Desempeño del reactor biológico híbrido}

Una vez seleccionados los polímeros que presentaron mayor eficiencia en las pruebas de inmovilización de biomasa (espuma de poliuretano, EPU y polibutadieno, BD) se procedió a realizar el montaje y operación del RBH. Los resultados de las pruebas realizadas se relacionan en la Tabla 3. Los ensayos fueron desarrollados con flujo continuo, empleando un tiempo de residencia hidráulica (TRH) de 2,6h, y una carga orgánica volumétrica de $2,7 \mathrm{Kg}$ DQO $\mathrm{m}^{-3} \mathrm{~d}^{-1}$.

Tabla 3. Eficiencia de remoción de materia orgánica y parámetros determinados en el efluente del RBH a los 8 y 13 días de operación.

\begin{tabular}{ccccccc}
\hline \multirow{2}{*}{ Prueba } & Día & T $\left({ }^{\circ} \mathrm{C}\right)$ & pH & SDT $\left(\mathrm{mgL}^{-1}\right)$ & \multicolumn{2}{c}{ Remoción $(\%)$} \\
\cline { 6 - 7 } Control & 8 & 27,1 & 7,6 & 283 & DBO & DQOt \\
& 13 & 26,9 & 7,5 & 218 & 71,8 & 60,9 \\
\multirow{2}{*}{ BD } & 8 & 27,5 & 7,1 & 193 & 89,1 & 57,9 \\
& 13 & 26,9 & 7,5 & 240 & 84 & 84,8 \\
\multirow{2}{*}{ EPU } & 8 & 29,5 & 7,4 & 243 & 93,8 & 87,4 \\
& 13 & 28,8 & 7,7 & 195 & 94,4 & 93,6 \\
\hline
\end{tabular}

DBO: demanda bioquímica de oxígeno; DQOt: demanda química de oxígeno total, BD: polibutadieno; EPU: espuma de poliuretano.

Como puede evidenciarse, al aplicar EPU como material soporte fueron obtenidos los mayores índices de remoción de materia orgánica a los 13 días de operación $(94 \%$ de remoción de la DQO total del sistema). Estos niveles de eficiencia fueron mayores respecto a los obtenidos con BD y con el tratamiento control, el cual fue operado solo con biomasa en suspensión (Tabla 3). Los demás parámetros determinados, tales como temperatura, $\mathrm{pH}$ y concentración de sólidos disueltos totales, presentaron valores similares en los tres tratamientos a los 8 y 13 dias de operación.

Los valores medios de $\mathrm{pH}$ determinados durante el tiempo de operación del sistema fueron de 7,4 $\pm 0,3$ (control); 7,4 $\pm 0,2$ (BD) y 7,5 $\pm 0,2$ (EPU), indicando el mantenimiento de valores cercanos a la neutralidad en los tres tratamientos. El perfil de variación de este parámetro se encuentra relacionado en la Figura 3a. Los valores medios de los sólidos 
disueltos totales (SDT), en $\mathrm{mgL}^{-1}$ fueron de 291,9 $\pm 69,3 ; 248,4 \pm 63,0$ y $270,2 \pm 73,7$, para el ensayo control, y sistemas con BD y EPU, respectivamente.

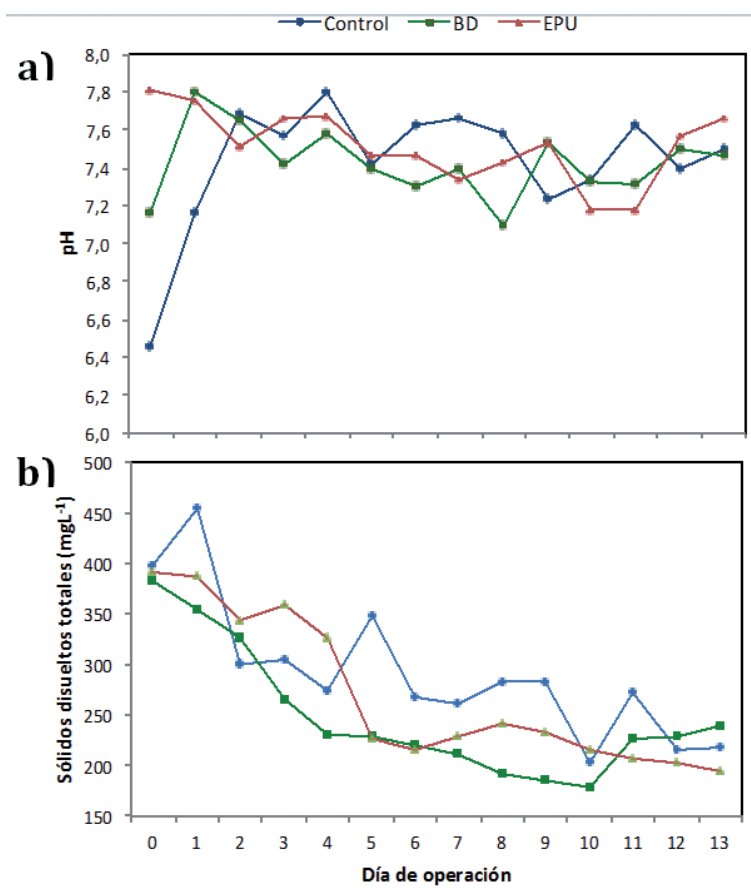

Figura 3. Parámetros determinados en el efluente del RBH para el ensayo control (biomasa en suspensión), y con incorporación de butadieno (BD) y espumas de poliuretano (EPU). a) Perfil de variación del pH; b) Perfil de variación de los sólidos disueltos totales.

Los niveles un poco superiores para este parámetro en el tratamiento control son debidos, fundamentalmente, a una mayor concentración de biomasa en suspensión en el afluente. Por otra parte, la temperatura de operación de los sistemas evaluados se encontró dentro de rango mesofílico $\left(27,5 \pm 1^{\circ} \mathrm{C}\right)$. Adicionalmente fue determinada la conductividad del efluente del sistema durante el tiempo de operación, encontrando valores medios (en $\mathrm{mS}$ ) de $573 \pm 137 ; 494 \pm 122$ y $530 \pm 134$, para el control, BD y EPU, respectivamente.

\section{Discusión}

La selección del material soporte para retención de biomasa metabólicamente activa constituye un aspecto relevante dentro de la operación y eficiencia de sistemas híbridos configurados para el tratamiento de aguas residuales. En este trabajo fueron evaluados cinco soportes, conformados por polímeros sintéticos de fácil adquisición. Con la finalidad de evitar interferencias en cuanto a diferencias en las condiciones de flujo de transferencia de masa y patrones hidrodinámicos, los polímeros fueron escogidos atendiendo a un patrón de similitud de tamaño y peso. La elección de los mejores materiales soporte para el sistema híbrido fue realizada en ensayos establecidos en proceso discontinuo, empleando caldo Luria Bertani (LB), y estimando la densidad bacteriana retenida. Para ello, fue seleccionado como modelo de biomasa un aislado bacteriano ambiental (Bacillus sp. H22), recuperado a partir de un humedal natural (14), el cual, según los resultados obtenidos, permaneció viable y metabólicamente activo durante los ensayos realizados, como fue observado directamente al estimar el crecimiento microbiano, Figura 2, e indirectamente, al realizar la estimación de los niveles de remoción de materia orgánica, Tabla 3. El perfil cinético determinado a partir de la biomasa en suspensión permitió inferir el tiempo necesario para alcanzar el final de la fase de crecimiento exponencial $(t=12 \mathrm{~h})$, periodo después del cual fue realizada la cuantificación de la biomasa adherida a cada soporte, encontrando entre $10^{5}$ a $10^{7}$ unidades formadoras de colonia por gramo de soporte, Tabla 2. Esta densidad puede aumentar progresivamente en función del tiempo de proceso y desarrollo de la biopelícula. No obstante, una vez alcanzada la fase estacionaria, fue evidenciada una diferencia significativa entre la cantidad de biomasa adherida por el butadieno (BD) y la espuma de poliuretano (EPU), Tabla 2, en comparación a los demás soportes evaluados. $\mathrm{Al}$ respecto, diversas investigaciones han puesto de manifiesto el potencial de estos dos materiales en la generación de biopelículas estables. Stephenson y colaboradores (16), en 2013, evaluaron BD y otros cuatro materiales como soportes a emplear en el desarrollo de una biopelícula con propiedades nitrificantes. En dicho estudio fue concluido que el BD permitió una retención de biomasa de 
hasta de 47,5g por $\mathrm{m}^{2}$ de material. Así mismo, ha sido establecido que la EPU constituye un medio soporte ideal para el crecimiento microbiano, al presentar alta porosidad y buena fuerza mecánica (16). La EPU, adicionalmente, permite el desarrollo de biomasa bajo diversos patrones de inmovilización, durante el tratamiento un variado tipo de efluentes $(12,17,18)$.

Dado que el BD y la EPU presentaron el mejor desempeño en las pruebas de adherencia celular, fueron seleccionados para la operación del RBH. En la Tabla 3 se observa el desempeño de los soportes, en términos de remoción de materia orgánica, representada como DBO y DQO total. Los ensayos realizados permitieron establecer una clara diferencia entre el nivel de remoción establecido como control, el cual fue realizado solo con biomasa suspendida, y los ensayos realizados en sistema híbrido, empleando BD y EPU como medios soportes. Tras estas determinaciones, fue establecido que el sistema híbrido presentó mayor eficiencia, alcanzando niveles de remoción a los 13 días de proceso, de hasta $84,0 \%$ y $78,4 \%$ de DBO y DQO total, para $\mathrm{BD}$, y de $94,4 \%$ y $93,6 \%$ de $\mathrm{DBO}$ y $\mathrm{DQO}$ total, respectivamente, para EPU, Tabla 3. Estos resultados fueron similares a los obtenidos por Peña y colaboradores en 2013 (19), los cuales emplearon un sistema CSTR con similar configuración a la empleada en este estudio, pero empleando biomasa en suspensión, alcanzando una remoción de DBO de hasta $92,5 \%$. No obstante, en dicho estudio fue aplicada una carga orgánica volumétrica de solo $0,5 \mathrm{Kg}$ DQO m $\mathrm{m}^{-3} \mathrm{~d}^{-1}$, siendo menor a la aplicada en

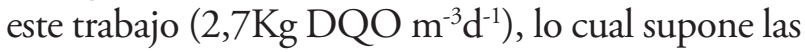
ventajas del uso del sistema híbrido en el aumento de carga proporcionada, manteniendo alta eficiencia de remoción de materia orgánica.

Los dos sistemas híbridos establecidos con BD y EPU mantuvieron niveles de $\mathrm{pH}$ cercanos a la neutralidad (entre 7,0 y 7,8, Figura 3a). Adicionalmente fue evidenciada la remoción de sólidos disueltos totales, encontrando valores medios inferiores en el efluente de los montajes híbridos, respecto al ensayo control, Figura 3b, lo cual puede presentar ventajas en términos de reducción en la generación de lodos por parte del sistema. El mejor desempeño de la EPU en el proceso propuesto concuerda con las observaciones realizadas por otros autores. Jianlong y colaboradores en 2000 (6), estudiaron el efecto de la aplicación de altas cargas orgánicas volumétricas de aguas residuales domésticas en un sistema híbrido, encontrando que el incremento de la biomasa estuvo directamente relacionado con el aumento de los niveles de remoción de DQO. Chu y Wang, en 2011(19-23), determinaron el potencial de la EPU en el desarrollo de biomasa en un reactor de lecho en movimiento, evidenciando hasta $0,067 \mathrm{~g}$ de biomasa adherida en cada gramo de material soporte, favoreciendo la remoción de DQO y nitrógeno amoniacal de aguas residuales domésticas con baja relación $\mathrm{C} / \mathrm{N}$.

Empleando otra configuración, Silva y colaboradores (6) en 2006 evaluaron la adhesión de biomasa (bacterias sulfato reductoras y arqueas metanogénicas) a EPU, carbón vegetal, polietileno de baja densidad y cerámica basada en alúmina. En dicho estudio fue concluido que el material que presentó mejor desempeño fue la EPU, tanto para la reducción de sulfatos, DQO y capacidad de retención celular, seguido por el carbón vegetal.

Finalmente, teniendo en cuenta los resultados obtenidos en este estudio, se concluye que el soporte constituido por espuma de poliuretano (EPU) presenta potencial aplicación en la operación de sistemas híbridos tipo $\mathrm{RBH}$, para tratamiento de aguas residuales domésticas, posibilitando mantener estabilidad operacional y alcanzando altos niveles de remoción de materia orgánica.

\section{Agradecimientos}

Los autores agradecen a la Fundación Universitaria de San Gil (UNISANGIL), sede Yopal (Casanare, Colombia), por el soporte financiero brindado a este trabajo. 


\section{Referencias}

1. Liu Y-Q, Moy BY-P, Tay J-H. COD removal and nitrification of low-strength domestic wastewater in aerobic granular sludge sequencing batch reactors. Enzyme Microb Technol. 2007;42(1):23-8.

2. Ahn Y-H. Sustainable nitrogen elimination biotechnologies: A review. Process Biochem. 2006;41(8):1709-21.

3. Chan YJ, Chong MF, Law CL, Hassell DG. A review on anaerobic-aerobic treatment of industrial and municipal wastewater. Chem Eng J. 2009;155(1-2):1-18.

4. Jianlong W, Hanchang S, Yi Q. Wastewater treatment in a hybrid biological reactor (HBR): Effect of organic loading rates. Process Biochem. 2000;36(4):297-303.

5. Kariminiaae-Hamedaani H-R, Kanda K, Kato F. Wastewater treatment with bacteria immobilized onto a ceramic carrier in an aerated system. J Biosci Bioeng. 2003;95(2):128-32.

6. Silva AJ, Hirasawa JS, Varesche MB, Foresti E, Zaiat M. Evaluation of support materials for the immobilization of sulfate-reducing bacteria and methanogenic archaea. Anaerobe. 2006;12(2):93-8.

7. Kourkoutas Y, Bekatorou A, Banat I., Marchant R, Koutinas A. Immobilization technologies and support materials suitable in alcohol beverages production: a review. Food Microbiol. 2004 Aug;21(4):377-97.

8. Leenen EJTM, Dos Santos VAP, Grolle KCF, Tramper J, Wijffels R. Characteristics of and selection criteria for support materials for cell immobilization in wastewater treatment. Water Res. 1996;30(12):2985-96.

9. Dong Z, Lu M, Huang W, Xu X. Treatment of oilfield wastewater in moving bed biofilm reactors using a novel suspended ceramic biocarrier. J Hazard Mater. 2011;196:123-30.

10. Osorio F, Hontoria E. Wastewater treatment with a doublelayer submerged biological aerated filter, using waste materials as biofilm support. J Environ Manage. 2002;65(1):79-84.

11. Chernicharo CA de L. Anaerobic Reactors. Aptara, editor. London: IWA; 2007. 188 p.

12. Guo W, Ngo H-H, Dharmawan F, Palmer CG. Roles of polyurethane foam in aerobic moving and fixed bed bioreactors. Bioresour Technol. 2010;101(5):1435-9.

13. An T, Zhou L, Li G, Fu J, Sheng G. Recent Patents on Immobilized Microorganism Technology and Its Engineering Application in Wastewater Treatment. Recent Patents Eng. 2008;2(1):28-35.
14. Botello Suárez WA, Puerto D, Montes D, Rodas EF. Detección y evaluación del potencial nitrificante de aislados bacterianos asociados a la rizósfera de tres especies de macrófitas. Rev Colomb Cienc Anim. 2014;6(2):414-23.

15. APHA, Awwa, WEF. Standard methods for the examination of water and wastewater. 21th ed. Association APH, Association AWW, Water Environment Federation, editors. Washington, DC: American Public Health Association; 2005.

16. Kim D, Kim K-Y, Ryu H-D, Min K-K, Lee S-I. Long term operation of pilot-scale biological nutrient removal process in treating municipal wastewater. Bioresour Technol. 2009; 100(13):3180-4.

17. Tan C, Ma F, Li A, Qiu S, Li J. Evaluating the Effect of Dissolved Oxygen on Simultaneous Nitrification and Denitrification in Polyurethane Foam Contact Oxidation Reactors. Water Environ Res. 2013;85(3):195-202.

18. Prabhavathi P, Rajendran R, Karthiksundaram S, Pattabi S, Dinesh Kumar S, Santhanam P. Enhanced bioremediation efficiency of denim industrial effluent using bacterial biofilm onto polyurethane matrix (review). Appl Biochem Microbiol. 2014;50(6):554-62.

19. Chu L, Wang J. Comparison of polyurethane foam and biodegradable polymer as carriers in moving bed biofilm reactor for treating wastewater with a low $\mathrm{C} / \mathrm{N}$ ratio. Chemosphere. 2011;83(1):63-8.

20. De Navia SLÁ, Torres SME. Calidad sanitaria del agua del Parque Natural Chicaque. Nova. 2013;11(20).

21. Ramírez LCC, Arévalo GZY, Moreno BVE. Solubilización de fosfatos: una función microbiana importante en el desarrollo vegetal. Nova. 2014; 12(21).

22. Galvez ZYA, Burbano VEM. Bacillus: género bacteriano que demuestra ser un importante solubilizador de fosfato. NOVA Publicación en Ciencias Biomédicas. 2015;12(22):165-77.

23. Ramírez LCC, Leal LCS, Rodríguez FAE. Determinación de la presencia de bacterias patógenas para el humano en aguas de riego en la cuenca alta de la sabana de Bogotá; DC Colombia. Nova. 2014;12(22). 